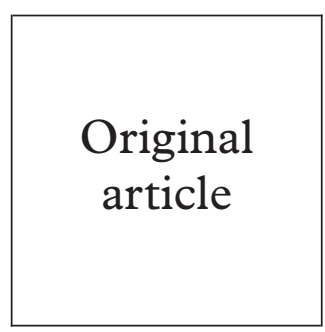

\title{
Risk factors for the acquisition of genital warts: are condoms protective?
}

\author{
L M Wen, C S Estcourt, J M Simpson, A Mindel
}

Objectives: To characterise risk factors for the acquisition of genital warts and specifically to determine whether condoms confer protection from infection.

Methods: A retrospective case-control study comparing demographic, behavioural, and sexual factors in men and women with and without newly diagnosed genital warts, who attended Sydney Sexual Health Centre (SSHC), an inner city public sexual health centre, in 1996. Data were extracted from the SSHC database. Crude odds ratios (OR) were calculated to compare cases and controls and significant factors were then controlled for using multivariate logistic regression to obtain adjusted odds ratios (ORs).

Results: 977 patients with warts and 977 controls matched by sex and date of attendance were included. In both sexes, univariate analysis revealed that younger age, more lifetime sexual partners, failure to use condoms, greater cigarette smoking and alcohol consumption were associated with warts, and there was a negative association with previous infection with Chlamydia trachomatis, Neisseria gonorrhoeae, hepatitis B, and genital herpes. In males, on multivariate analysis, factors which remained significant were younger age, more lifetime sexual partners; failure to use condoms, greater cigarette smoking, and previous chlamydia. In women, factors which remained significant were younger age, more lifetime sexual partners, condom use, marital status, and previous infections with Chlamydia trachomatis and herpes.

Conclusions: Independent risk factors for genital warts include younger age, greater number of lifetime sexual partners, and smoking. Consistent condom use significantly reduces the risk of acquiring genital warts.

(Sex Transm Inf 1999;75:312-316)

Keywords: genital warts; risk factors; condoms

\section{Introduction}

Genital warts are increasing in incidence worldwide and, as a consequence of the control of the bacterial STDs, have become one of the most common infections in the developed world. The Centers for Disease Control (CDC) estimated that there were between 500000 and one million new cases of genital warts diagnosed in STD clinics in the United States in $1992 .{ }^{1}$ In the past decade, there has been about a $300 \%$ increase in the number of patients with genital warts who attend STD clinics in the United Kingdom. ${ }^{2}$ In Australia, the Sydney Sexual Health Centre (SSHC), one of the largest public STD clinics, has reported a sharp rise in genital warts during the past 20 years. The proportion of patients attending SSHC with genital warts annually has increased from $1 \%$ in 1973 to $7 \%$ in recent years. ${ }^{3}$ It is the commonest diagnosis in the clinic. A similar increase in cases has been reported in other countries. ${ }^{45}$ There are several possible reasons for this increase, including changes in sexual behaviour, subclinical human papillomavirus (HPV) infection, inadequate treatment, and frequent relapses.

The identification of risk factors for the acquisition of HPV is complicated by the high rate of subclinical infection, the absence of routine diagnostic tests to establish diagnosis, and the use of different testing methods in published studies. Previous studies have suggested a possible link between HPV infection and a number of demographic and sexual fac- tors, including younger age, greater number of sexual partners, use of oral contraceptives, and smoking. ${ }^{6-10}$ However, results from different studies have failed to confirm these as risk factors. ${ }^{7-11}$ Other factors such as race, socioeconomic status, and marital status have been investigated in some studies, but again, the results have been inconclusive.

This case-control study tested the association between a variety of potential risk factors and the presence of genital warts in clinic attenders, using a multivariate model to determine independent risk factors.

\section{Subjects and methods}

All patients with a new diagnosis of macroscopic genital warts who attended SSHC from 1 January to 31 December 1996 were included in the study group. Controls were patients who were not diagnosed as having genital warts, matched by sex and same date of attendance. Patients with cervical intraepithelial neoplasia (CIN) and HPV (cytological diagnosis) were excluded since these diseases are related to HPV infection but may have different transmission characteristics and natural histories.

Data were obtained from computerised pro forma medical records held on the clinic database. Continuous variables, such as age, were treated as categorical variables by grouping. Comparisons between cases and controls were performed using $\chi^{2}$ tests for categorical variables, using the Mantel-Haenszel trend test when appropriate. Crude odds ratios with their 
Table 1 Risk of genital warts in relation to condom use

\begin{tabular}{|c|c|c|c|}
\hline Condom use & $\begin{array}{l}\text { Cases } \\
\text { No (\%) }\end{array}$ & $\begin{array}{l}\text { Controls } \\
\text { No (\%) }\end{array}$ & $\begin{array}{l}\text { Adjusted OR } \\
(95 \% \mathrm{CI})^{\star}\end{array}$ \\
\hline \multicolumn{4}{|l|}{ Ment } \\
\hline$\chi_{4}^{2}=62.3,<0.001$ & & & \\
\hline Not applicable, no sex & $78(13.0)$ & $52(11.6)$ & 1 \\
\hline Never & $195(32.4)$ & $80(17.9)$ & $1.9(1.3-2.9)$ \\
\hline Sometimes < $50 \%$ & $89(14.8)$ & $41(9.2)$ & $3.0(2.2-4.3)$ \\
\hline Usually >50\% & $81(13.5)$ & $52(11.6)$ & $0.9(0.7-2.0)$ \\
\hline Always $(100 \%)$ excluding breakages & $159(26.4)$ & $222(49.4)$ & $\begin{array}{l}0.7(0.3-0.9) \\
\mathrm{p}<0.0001\end{array}$ \\
\hline \multicolumn{3}{|l|}{ Women $\ddagger$} & \\
\hline Not applicable, no sex & $34(10.8)$ & $23(9.9)$ & 1 \\
\hline Never & $91(28.9)$ & $63(27.0)$ & $1.8(0.9-3.6)$ \\
\hline Sometimes $<50 \%$ & $59(18.7)$ & $22(9.4)$ & $1.7(1.0-2.9)$ \\
\hline Usually > $50 \%$ & $54(17.1)$ & $38(16.3)$ & $0.8(0.7-1.7)$ \\
\hline Always $(100 \%)$ excluding breakages & $77(24.4)$ & $87(37.3)$ & $\begin{array}{l}0.7(0.4-1.0) \\
\mathrm{p}=0.013\end{array}$ \\
\hline
\end{tabular}

${ }^{\star}$ Multivariate logistic regression with variables shown to be associated with genital warts in univariate analysis:

†Age group, number of lifetime sex partners, and smoking; łage group, marital status, and occupations.

$\mathrm{OR}=$ odds ratio, $\mathrm{CI}=$ confidence interval

95\% confidence interval (CI) were calculated in a univariate analysis to compare grouped study factors between cases and controls.

Factors which were related to genital warts in univariate analysis $(\mathrm{p}<0.10)$ were further analysed using unconditional logistic regression models to permit statistical control of confounding variables. These included demographic characteristics, number of sexual partners, smoking, and alcohol consumption. The adjusted odds ratios (ORs) with their 95\% CI were then calculated. The use of OR as an approximate relative risk (RR) is justified since genital warts are considered to be a rare disease in the general population. All analyses were conducted separately for women and men. Statistical analysis was performed using the sPss package.

\section{Results}

A total of 12496 individuals (7234 men, 5262 women) attended Sydney Sexual Health Centre during 1996 and 977 patients (649 men and 328 women) presented with a new diagnosis of genital warts.

The sample was ethnically mixed and the countries of birth of cases and controls were similar. Individuals were predominantly Australian born residents $(61.3 \%)$. The rest were from England (10.5\%), New Zealand (6.5\%), and 60 other countries around the world. Most of the subjects were sexually experienced. The median lifetime number of sex partners was 20 for men and eight for women. Only $3.6 \%$ of cases and $6.7 \%$ of controls reported no sex partners in their lifetime.

The median age of men and women with genital warts was 28 years and 24 years respectively compared with 33 years and 30 years for their corresponding controls.

On univariate analysis, the presence of genital warts was associated with a number of demographic, social, and sexual factors. In males these included younger age, any history of cigarette smoking, alcohol consumption, greater number of lifetime sexual partners, and less frequent condom use. In females, risk factors included younger age, never having married, being unemployed, cigarette smoking, alcohol consumption, and less frequent condom use. In men a previous history of chlamydia and gonorrhoea was negatively associated with the presence of genital warts with unadjusted ORs of 0.5 (95\% CI 0.4-0.6) and 0.5 (95\% CI 0.4-0.8) respectively. In women a previous history of chlamydia and gonorrhoea was also negatively associated with the presence of genital warts with unadjusted ORs of $0.5(95 \%$ CI $0.3-0.8)$ and 0.3 (95\% CI $0.1-0.7)$ respectively. In addition, for women, a negative association was noted for previous genital herpes (unadjusted OR $0.4,95 \%$ CI $0.2-0.7$ ) and previous hepatitis B (unadjusted OR 0.3, 95\% CI 0.1-0.7).

A highly significant dose-response effect was seen for cigarette smoking. Smokers of more than 10 cigarettes per day were twice as likely to have genital warts as were non-smokers (unadjusted $\mathrm{OR}=2.0,95 \%$ CI $1.7-3.7$ ), while smokers of less than 10 cigarettes per day showed a significant but lesser risk (OR 1.5, 95\% CI 1.1-2.1). The results were similar for women.

Alcohol consumption in men was associated with genital warts and also displayed a significant dose-response effect $\left(p_{\text {trend }}=0.002\right)$. Compared with men who drank no alcohol, the OR of genital warts for men who consumed more than $51 \mathrm{~g}$ per week of alcohol was 1.5 with $95 \%$ CI of 1.1 to 2.0 . In contrast, no significant difference in alcohol consumption between cases and controls was found for women.

The results of multivariate analysis are shown in tables 1 and 2 .

The difference in age distribution between those with and without genital warts remained significant for both men and women, and the odds ratio showed a very strong negative trend with age (table 2). In men, the effect of smoking and number of lifetime partners was minimally affected by adjustment for other variables. However, alcohol consumption was not independently associated with increased risk of acquisition of genital warts.

In contrast, in women, after controlling for confounding variables, smoking, and number of lifetime sexual partners were no longer significantly associated with genital warts. Alcohol remained marginally significant. Compared with women who drank no alcohol, the adjusted odds ratio for genital warts for women who drank more than $51 \mathrm{~g}$ of alcohol per week was 1.2 (95\% CI 0.8-1.9). Interestingly, the effect of marital status remained highly significant but the direction of the OR reversed. The relative risk of genital warts for women who had separated or divorced was around three times higher with $95 \%$ CI of 1.5-6.0, compared with those women who had never married. Occupation remained a significant risk factor.

After adjusting for other confounders, the difference in condom use between cases and controls remained significant for both men and women (table 1). Consistent condom use conferred significant reduction in risk of acquisition of warts. 
Table 2 Risk factors for genital warts in men and women

\begin{tabular}{|c|c|c|c|}
\hline Variables & $\begin{array}{l}\text { Cases } \\
\text { No (\%) }\end{array}$ & $\begin{array}{l}\text { Controls } \\
\text { No }(\%)\end{array}$ & $\begin{array}{l}\text { Adjusted OR } \\
(95 \% \mathrm{CI})^{*}\end{array}$ \\
\hline \multicolumn{4}{|l|}{ Ment } \\
\hline \multicolumn{4}{|c|}{ Age (years) $\chi_{4}^{2}=104.0, \mathrm{p}<0.0001$} \\
\hline $16-19$ & $11(1.7)$ & $2(0.3)$ & 1 \\
\hline $20-24$ & $140(21.6)$ & $63(9.7)$ & $0.4(0.1-1.7)$ \\
\hline $25-29$ & $226(34.8)$ & $143(22.0)$ & $0.2(0.1-1.1)$ \\
\hline $30-34$ & $132(20.3)$ & $160(24.7)$ & $0.1(0-0.6)$ \\
\hline$\geqslant 35$ & $140(21.6)$ & $281(43.3)$ & $0.1(0 .-0.4)$ \\
\hline \multirow{2}{*}{\multicolumn{4}{|c|}{ Sex partners in lifetime $\chi_{4}^{2}=15.66, p=0.003$}} \\
\hline & & & \\
\hline $0-1$ & $30(4.6)$ & $58(9.2)$ & 1 \\
\hline $2-4$ & $37(5.7)$ & $50(7.9)$ & $1.1(0.5-2.1)$ \\
\hline $5-10$ & $138(21.3)$ & $128(20.2)$ & $1.8(1.0-3.1)$ \\
\hline $11-20$ & $165(25.4)$ & $129(20.4)$ & $2.1(1.2-3.6)$ \\
\hline$\geqslant 21$ & $279(43.0)$ & $268(42.3)$ & $2.1(1.3-3.5)$ \\
\hline & & & $\mathrm{p}_{\text {trend }}=0.004$ \\
\hline \multicolumn{4}{|c|}{ Cigarettes per day $\chi_{2}^{2}=26.3, p<0.001$} \\
\hline 0 & $385(58.8)$ & $453(71.8)$ & 1 \\
\hline $1-10$ & $112(17.3)$ & $89(14.1)$ & $1.3(1.0-1.8)$ \\
\hline \multirow[t]{2}{*}{$\geqslant 11$} & $154(23.9)$ & $89(14.1)$ & $1.9(1.0-2.3)$ \\
\hline & & & $\mathrm{p}_{\text {trend }}=0.001$ \\
\hline \multicolumn{4}{|l|}{ Women $\dagger$} \\
\hline \multicolumn{4}{|c|}{ Age (years) $\chi_{4}^{2}=118.1, \mathrm{p}<0.0001$} \\
\hline $16-19$ & $38(11.6)$ & $14(4.3)$ & 1 \\
\hline $20-24$ & $140(42.7)$ & $54(16.5)$ & $0.9(0.4-1.8)$ \\
\hline $25-29$ & $98(29.9)$ & $86(26.2)$ & $0.3(0.2-0.7)$ \\
\hline $30-34$ & $27(8.2)$ & $66(20.1)$ & $0.1(0-0.2)$ \\
\hline$\geqslant 35$ & $25(7.6)$ & $108(32.9)$ & $0 \quad(0-0.1)$ \\
\hline & & & $\mathrm{p}_{\text {trend }}<0.0001$ \\
\hline \multicolumn{4}{|c|}{ Sex partners in lifetime $\chi_{4}^{2}=17.86, p=0.001$} \\
\hline $0-1$ & $28(8.5)$ & $40(12.3)$ & 1 \\
\hline $2-4$ & $76(23.2)$ & $60(18.5)$ & $1.3(0.6-2.6)$ \\
\hline $5-10$ & $114(34.8)$ & $103(31.7)$ & $1.0(0.5-1.9)$ \\
\hline $11-20$ & $81(24.7)$ & $62(19.1)$ & $1.3(0.7-2.6)$ \\
\hline$\geqslant 21$ & $29(8.8)$ & $60(18.5)$ & $0.7(0.3-1.5)$ \\
\hline & & & $\mathrm{p}_{\text {trend }}=0.3$ \\
\hline \multicolumn{4}{|c|}{ Marital status $\chi_{2}^{2}=12.47, p=0.002$} \\
\hline Never married & $254(78.6)$ & $210(66.5)$ & 1 \\
\hline Married/defacto & $37(11.5)$ & $63(19.9)$ & $1.2(0.7-2.0)$ \\
\hline Separated/divorced & $32(9.9)$ & $43(13.6)$ & $3.0(1.5-6.0)$ \\
\hline \multicolumn{4}{|c|}{ Occupation $\chi_{5}^{2}=11.03, \mathrm{p}=0.05$} \\
\hline Unemployed & $43(13.3)$ & $28(8.8)$ & 1 \\
\hline Student & $48(14.5)$ & $63(19.5)$ & $0.4(0.2-0.8)$ \\
\hline Home duties & $4(1.2)$ & $10(3.1)$ & $0.8(0.2-3.9)$ \\
\hline Benefit & $10(3.1)$ & $8(2.5)$ & $2.6(0.8-8.8)$ \\
\hline Sex workers & $47(14.6)$ & $61(19.2)$ & $0.7(0.3-1.4)$ \\
\hline Employed & $171(52.9)$ & $149(46.9)$ & $1.1(0.6-2.1)$ \\
\hline
\end{tabular}

${ }^{\star}$ Multivariate logistic regression with variables shown to be associated with genital warts in univariate analysis.

†Each factor is adjusted for other factors in the group.

$\mathrm{OR}=$ odds ratio, $\mathrm{CI}=$ confidence interval.

Previous chlamydial infection in both sexes remained negatively associated with risk of genital warts on multivariate analysis. Individuals with a history of chlamydia had a lower risk of genital warts than those with no previous chlamydia. In men and women the OR was 0.52 with $95 \%$ CI of $0.4-0.7$, and $0.3-0.9$ respectively. In addition, women who had genital herpes had a lower risk of acquisition of genital warts, compared with controls (OR 0.53 with $95 \%$ CI $0.3-1.0$ ).

\section{Discussion}

This study aimed to address some of the inconsistencies in published reports by using a retrospective case-control design with almost 1000 patients who presented to a sexual health clinic with exophytic genital warts. Controls were from the same population and matched for sex and day of attendance. Analyses were conducted separately for men and women because risk factors differed between the sexes. A multivariate model was used to establish independent variables.

Our study design did not allow for the control of all potential confounding variables.
Data were extracted retrospectively from the pro forma medical records, which are in routine use in the clinic. It was not possible to obtain information on certain factors of potential relevance, such as oral contraceptive use, age at first intercourse, patients' knowledge of HPV infection, nature of genital skin contact, and specific sexual practice, as this information is not collected in a standardised format. The diagnosis of genital warts was made by clinical impression and not all patients were seen by the same physician. This may result in misdiagnosis. In common with all studies on sexual behaviour, recall bias with respect to numbers of sexual partners and condom use cannot be excluded. Our study sample was confined to clinic attenders. It is possible that these patients are not representative of patients with warts in the general community and that this introduced selection bias.

The use of condoms to reduce risk of acquisition of genital warts is often advised in clinical practice, ${ }^{12}$ as it is believed that HPV is transmitted by skin to skin contact and that condoms provide a barrier to transmission of HPV from infected areas of skin. However, we are not aware of any published studies which have established that the use of condoms does reduce the transmission of HPV for patients with genital warts. In contrast, previous studies have failed to show a protective effect of condom use. ${ }^{13-15}$ This study is the first to demonstrate that in both sexes, failure to use condoms is independently associated with an increased risk of acquisition of genital warts, and that consistent condom use is associated with a decreased risk of acquisition of genital warts (table 1). These data go far in answering important questions in the demand for an evidence based approach ${ }^{12}$ to patient information on transmission of genital warts and methods of risk.

In men and women, genital warts were independently associated with younger age, which supports data from previous studies and coincides with the time of peak prevalence of HPV. ${ }^{16}{ }^{17}$ The peak incidence in women occurred in the early 20 s and in men in the late 20s. This pattern was similar to but slightly later than in Simms and Fairley's large UK study, which reported the peak attendance for first episode genital warts in women to be in the 16-24 year age group and in men in the 20-24 year age group. ${ }^{18}$ Genital warts decreased markedly with increasing age, independent of sexual behaviour and numbers of sexual partners. The reasons for this age effect are unclear but may be related to the host genital tract immune response. The importance of host responses in determining wart clearance is well recognised. ${ }^{1920}$ It has been suggested that older individuals may be more resistant to infection, may be able to clear virus rapidly, or induce faster wart regression. ${ }^{21}$

Greater numbers of lifetime sexual partners were independently associated with an increased risk of genital warts in men only. Men with 10 or more lifetime partners were approximately twice as likely to acquire genital warts as were men with no or one lifetime sexual 
partner. A significant trend was seen for increasing risk of genital warts with increasing numbers of lifetime sexual partners. It is interesting that no such association was found for women in our study. Data from published studies have linked risk of acquisition of genital warts with multiple sexual partners for both sexes. ${ }^{78}$ However, studies of HPV DNA detection from the genital tract have yielded inconsistent results. In part this could be due to differences in sensitivities and specificities of the particular molecular technique used, but also to the differing age and STD profiles of the populations under study. In one study of 13-19 year old sexually active women, a history of more than three previous sexual partners was the most important risk factor for HPV DNA detection. ${ }^{22}$ However, a number of other studies have failed to detect an association between numbers of lifetime sexual partners and HPV detection. ${ }^{21} 23$

In this study, cigarette smoking was strongly linked to the risk of genital warts in men. A dose-response effect was detected in which smokers of more than 10 cigarettes per day were found to be twice as likely to have genital warts as non-smoking men. In contrast, in women no such association was detected after adjusting for other risk factors. The reasons for such a striking sex difference require further study. A recent study of the relation between cigarette smoking and the risk of acquisition of genital warts in a cohort of HIV positive women and a group of HIV negative female controls, reported that in both groups, smokers were five times as likely to develop genital warts as were non-smokers, and that HIV positive women were significantly more likely to develop warts than the negative controls. ${ }^{24}$ The baseline prevalence of HPV DNA was similar in smokers and non-smokers. This suggests that the rate of progression of exophytic genital warts is increased in smokers and may reflect a local cigarette induced immune modulation. However, a study in Greenland and Denmark did not show any association between smoking and HPV infection. ${ }^{23}$

Few studies have examined the effect of marital status and occupation on prevalence of genital warts. In this study, after adjustment for confounding variables, divorced or separated women were around three times more likely to acquire genital warts as women who had never married. One previous study reported that unmarried women were more at risk of HPV infection. ${ }^{21}$ In that report, the subjects were young women only, which makes it difficult to relate the results to our study which included women of a much wider age range. An early case-control study of 120 women with condylomata found that patients were more likely to be single or divorced than the controls, ${ }^{25}$ in partial agreement with our results. Low socioeconomic status has been implicated as a risk factor for HPV infection in some countries. ${ }^{6}$ In this study, women receiving benefit were most likely to have genital warts. It is unclear why no such associations were detected in men.

Men and women who reported previous chlamydial infection and women who had a history of genital herpes were less likely to acquire genital warts. In contrast, Beuret et al reported that women with genital warts were more likely to have evidence of previous chlamydial infection by serum antibody testing. ${ }^{25}$ In addition, Davidson et al hypothesise that presence of other STDs, particularly genital herpes, may increase the risk of acquisition of HPV in the genital tract. ${ }^{14}$ Interpretation of our findings is limited by the retrospective nature of the study and further work is required to investigate this apparent association,

To our knowledge, this is the first published study which provides strong evidence for the protective role of condoms in reducing the risk of acquisition of genital warts. Information currently given to patients by sexual health physicians on condom use in the prevention of transmission of genital HPV is highly variable. ${ }^{12}$ This paper provides the evidence upon which to base future condom recommendations.

We are grateful to Richard Rohrsheim for assistance with the database.

Contributors: L M Wen participated in protocol design, data

Contributors: L M Wen participated in protocol design, data
analysis, and interpretation, review of manuscript; C S Estcourt analysis, and interpretation, review of manuscript; C S Estcourt was involved in data interpretation and wrote the paper; J M
Simpson gave statistical advice in study design, was involved in data analysis including multivariate analysis and data interpretation, and review of manuscript; A Mindel had the original idea for the study, and participated in protocol design, data interpretation, and review of manuscript.

1 Centers for Disease Control and Prevention. Division of STD/HIV Prevention 1992 Annual Report. Atlanta, GA: US Department of Health and Human Services, Public Health Service Centers for Disease Control and Prevention, 1993.

2 Sonnex C. The clinical features of genital and perigenital human papillomavirus infection. In: Mindel A, ed. Genital warts, human papillomavirus infection. London: Edward Arnold, 1995

3 Donovan B, Mindel A. Are genital herpes and warts really a disappearing problem? Aust f Public Health 1995;19:216-7. disappearing problem? A ust f Public Health 1995;19:216-7. 4 Shao C, Liang G. STD epidemiology analysis at national
surveillance spots in the period 1987-1990. Chin Med Scif surveillance spot

5 Bundesamt FG. Sexual ubertragbare krankheiten nach auslandrisen 1994;Bull No 20:371-9.

6 Bauer HM, Hildesheim A, Schiffman MH. Determinants of genital human papillomavirus in low-risk women in Portland, Oregon. Sex Transm Dis 1993;20:74-8.

7 Karlsson R, Jonsson M, Edlund K, et al. Lifetime number of partners as the only independent risk factor for human papillomavirus infection: a population-based study. Sex Transm Dis 1995;22:19-27.

8 Wheeler CM, Parmenter CA, Hunt WC, et al. Determinants of genital human papillomavirus infection among cytologically normal women attending the University of New cally normal women attending the University of New Mexico

9 Ross JDC. Is oral contraceptive associated with genital warts? Genitourin Med 1996;72:330-3.

10 Daling JR, Sherman KJ, Weiss NS. Risk factors for condyloma acuminatum in women. Sex Transm Dis 1986;13:16-8

11 Hildesheim A, Gravitt P, Schiffman MH, et al. Determinants of genital human papillomavirus infection in low-income women in Washington, DC. Sex Transm Dis 1993;20:279-85

12 McClean HL, Hillman RJ. Anogenital warts and condom use-a survey of information given. Genitourin Med 1997;73:203-6.

13 Ley C, Bauer HM, Reingold A, et al. Determinants of genital human papillomavirus infection in young women. $\mathcal{F}$ Nat Cancer Inst 1991:83:997-1003.

14 Davidson M, Schnitzer PG, Bulkow LR, et al. The prevalence of cervical infection with human papillomavirus and cervical dysplasia in Alaska native women. F Infect Dis 1994;169:792-800.

15 Evans BA, Kell PD, Bond RA, et al. Heterosexual relationships and condom-use in the spread of sexually transmitted diseases to women. Genitourin Med 1995;71:291-4.

6 Daling JR, Weiss NS, Sherman KJ. History of genital warts in a selected population. Lancet 1984;1:157-8.

17 Melkert P W, Hopman E, van den Brule AJ, et al. Prevalence of HPV in cytomorphologically normal cervical smear, as determined by the polymerase chain reaction, is age dependent. Int $\mathcal{f}$ Cancer 1993;53:919-23.

18 Simms I, Fairley CK. Epidemiology of genital warts in England and Wales: 1971 to 1994 . Genitourin Med 1997;73: $365-7$.

19 Coleman N, Birley HD, Renton AM, et al. Immunological events in regressing warts. Am f Clin Pathol 1994;102:768-

20 Frazer IH. Immunology of papillomavirus infection. Curr Opin Immunol 1996;8:484-91 
21 Reeves WC, Brinton LA, Garcia M, et al. Human papillomavirus infection and cervical cancer in Latin America. N Engl f Med 1989;320:1437-41

22 Moscicki A-b, Palefsky J, Gonzales J, et al. Human papilloma virus infection in sexually active adolescen females: prevalence and risk factors. Paediatr Res 1990;28: 507-13.

23 Kjaer S K, de Villiers E M, Caglayan H, et al. Human papillomavirus, herpes simplex virus and other potential risk factors for cervical cancer in a high risk area (Greenland) and a low-risk area (Denmark)—a second look. Brf Cancer 1993;67:803-7.
24 Feldman JG, Chirgwin K, Dehovitz JA, et al. The association of smoking and risk of condyloma accuminatum in women. Obstet Gynecol 1997;89:346-50.

25 Beuret T, Sadoul G, Fari A, et al. Comparative epidemiologic study of 120 patients with condylomatous lesions and 120 control patients. F Gynecol Obstet Biol Reprod 1987;16: 555-64. 